\title{
A rare case of autoimmune pancytopenia (AIP) preceded by refractory anemia: possible involvement of cytomegalovirus (CMV) infection in pathogenesis
}

\author{
Nobuo Takemori ${ }^{1 *}$, Goro Imai ${ }^{2}$, Hirobumi Matsumura ${ }^{3}$, Toshio Fukuda ${ }^{4}$ and Masaru Kojima ${ }^{5}$ \\ ${ }^{1}$ Division of Hematology, Internal Medicine, Imai Hospital, Tanaka-cho 100, Ashikaga, Tochigi, 326-0822, Japan \\ ${ }^{2}$ Division of General Medicine, Internal Medicine, Imai Hospital, Tanaka-cho 100, Ashikaga, Tochigi, 326-0822, Japan \\ ${ }^{3}$ Division of Clinical Laboratory Medicine, Internal Medicine, Imai Hospital, Tanaka-cho 100, Ashikaga, Tochigi, 326-0822, Japan \\ ${ }^{4}$ Graduate School of Health Science, Gunma University, Maebashi, Gunma, 371-8514, Japan \\ ${ }^{5}$ Department of Anatomic and Diagnostic Pathology, Dokkyo Medical University School of Medicine, Mibu-machi, Shimotsuga-gun, Tochigi, 321-0293, Japan
}

\begin{abstract}
Autoimmune pancytopenia (AIP) is known to be caused by coexisting autoantibodies against three lineages of blood cells. We report a rare case of autoimmune pancytopenia (AIP) which was preceded by refractory anemia. An 85-year-old man with a 4 and half-year history of unexplained anemia presented in October 2016 because of remarkable pancytopenia and fever. Since direct and indirect anti-globulin tests as well as anti-platelet antibody were positive, Evans' syndrome was initially suspected. However, the patient also had remarkable neutropenia. Additional examination for anti-neutrophil antibody showed the presence of anti-neutrophil IgG antibody specific for human neutrophil alloantigen (HNA)-1a and HNA-2. A diagnosis of AIP was thus established. Subsequent steroid therapy was effective for improving thrombocytopenia as well as neutropenia; nevertheless, the improvement of anemia seemed insufficient. Furthermore, a transient thrombocytopenia appeared 2 months after admission during the treatment. This event in addition to a febrile episode and elevated levels of CRP, $\beta 2$-microglobulin and soluble interleukin-2 receptors seen at initial presentation prompted us to investigate if there had been specific infections by using the previously stocked serum samples. Consequently, the patient was found to have cytomegalovirus (CMV) infection; the presence of CMV-IgM antibody as well as CMV-DNA was demonstrated in the serum at the reappearance of thrombocytopenia. Since positive CMV-IgG and negative CMV-IgM were retrospectively confirmed in the stocked serum at initial presentation, it was considered that a reactivation of CMV occurred during the hospital course. The bone marrow aspirations carried out during hospitalization were compatible with refractory anemia, indicating that the patient already had refractory anemia before developing AIP.
\end{abstract}

The recognition of AIP should be a requisite for the correct diagnosis in some cases of Evans' syndrome associated with leukocytopenia. Special consideration should be given to the possible involvement of CMV infection in pathogenesis of autoimmune cytopenia(s) or AIP. Insufficient recovery from anemia seemed to be related with coexisting refractory anemia.

\section{Introduction}

In 1949, Evans and Duane [1] first reported the association of acquired autoimmune hemolytic anemia (AIHA) with thrombocytopenia and/or leukocytopenia. In 1951, they reported 4 cases of AIHA associated with primary thrombocytopenic purpura, and suggested a possible antibody-mediated autoimmune mechanism as the cause [2]. Subsequently, the coexistence of AIHA and autoimmune thrombocytopenia is usually referred to as Evans' syndrome. Recently, more reliable techniques for assaying anti-granulocytic antibodies had been established, and the presence of neutropenia caused by autoantibody against neutrophils has been documented. This disorder is referred to as autoimmune neutropenia (AIN) [3-6]. Afterwards, a number of composite cases of AIN, AIHA and autoimmune thrombocytopenia have been found and documented. Such a syndrome is referred to as idiopathic combined immunecytopenia [7], combined autoimmune cytopenia [8] or autoimmune pancytopenia (AIP) [9-11]. Most cases of AIP thus far reported were of secondary types which were related to allogenic bone marrow (BM) transplantation $[9,10]$.

Recently, we encountered a rare case of AIP preceded by refractory anemia in which cytomegalovirus (CMV) reactivation was demonstrated. In this paper, the importance of recognizing AIP in cases of Evans' syndrome associated with leukocytopenia is emphasized. The possible involvement of CMV infection in pathogenesis of unknown thrombocytopenia or AIP, and the relationship between AIP and refractory anemia are discussed.

\section{Case presentation}

An 85 -year-old man $(49 \mathrm{~kg} / 161 \mathrm{~cm})$ with a 4 and half-year history of unexplained anemia (red blood cell [RBC]: $3.31 \sim 3.91 \times 10^{12} / \mathrm{L}$, hemoglobin $[\mathrm{Hb}]: 10.8 \sim 12.6 \mathrm{~g} / \mathrm{dL}$ ) and hypertension since mid-fifties of age was referred to Imai Hospital from a family physician on October 25, 2016 because of oral cavity bleeding and remarkable pancytopenia.

Correspondence to: Nobuo Takemori, Division of Hematology, Internal Medicine, Imai Hospital, Tanaka-cho 100, Ashikaga, Tochigi, 326-0822, Japan, Tel: +81-276-76-7845; Fax: +81-276-76-7845; E-mail: takemori_nobuo@circus.ocn.ne.jp

Key words: AIP, CMV infection, Evans syndrome, HNA, refractory anemia, steroid therapy

Received: May 12, 2017; Accepted: May 25, 2017; Published: May 29, 2017 

pathogenesis

During 3 days before admission, the patient was febrile with sore throat. On admission, he had a temperature of $38^{\circ} \mathrm{C}$, and seemed pale and exhausted. There were several submucosal hemorrhages on the palate and hematomas on the lower lip and the left buccal mucosa. Multiple purpuras were noted over the surfaces of upper and lower extremities. Hepatosplenomegaly and superficial lymph node swellings were absent. His past history was somewhat complicated. He has been troubled with lumbar disc hernia since November 2015 and was previously treated with loxoprofen by a local orthopedic surgeon. In March 2016, acute renal failure associated with severe thrombocytopenia $\left(30 \times 10^{9} / \mathrm{L}\right)$, possibly caused by loxoprofen administration, developed. Both disorders were successfully treated with the hemodialysis for a short period of time in other hospital (the exact cause of thrombocytopenia remains unknown). On September 6, 2016, the patient received a laminectomy of 4 th and 5 th lumbar vertebrae in an orthopedic division of another hospital without receiving any blood transfusions. The postoperative course was uneventful (Figure 1).

Onadmission (October 25,2016), a completeblood cell count showed anemia (RBC count, $2.34 \times 10^{12} / \mathrm{L} ; \mathrm{Hb}, 7.3 \mathrm{~g} / \mathrm{dL}$; reticulocytes, $15.4 \%$ ), leukocytopenia (white blood cell [WBC] count, $1.1 \times 10^{9} / \mathrm{L}$ with $40 \%$ neutrophils, $24 \%$ lymphocytes, $32 \%$ monocytes, $3 \%$ eosinophils and $1 \%$ basophils) and thrombocytopenia (platelet count, $1.0 \times 10^{9} / \mathrm{L}$ ). Elevated levels of CRP $(9.73 \mathrm{mg} / \mathrm{dL}$; $\mathrm{nr} .0 \sim 0.26 \mathrm{mg} / \mathrm{dL})$, total bilirubin $(1.57 \mathrm{mg} /$ $\mathrm{dL}$; nr. $0.2 \sim 1.2 \mathrm{mg} / \mathrm{dL})$, indirect bilirubin $(1.3 \mathrm{mg} / \mathrm{dL}$; nr. $0.1 \sim 0.3 \mathrm{mg} /$ dL), LDH (255 IU/L; nr. 106-211 IU/L), BUN (23.8 mg/dL; nr. 8 20 $\mathrm{mg} / \mathrm{dL})$, soluble-IL-2 receptors (s-IL-2R) $(1,310 \mathrm{U} / \mathrm{mL}$; nr. 124 466 $\mathrm{U} / \mathrm{mL}$ ), $\beta 2$-microglobulin (3.3 mg/L; nr. 0.9 1.9 mg/L), ferritin (551 $\mathrm{ng} / \mathrm{mL}$; nr. 39.4 340 ng/mL) and non-specific IgE $(1,500 \mathrm{IU} / \mathrm{mL} ; \mathrm{nr}$. $\leq 170 \mathrm{IU} / \mathrm{mL})$, and reduced levels of serum Fe $(41 \mu \mathrm{g} / \mathrm{dL}$; nr. $54 \sim 181$ $\mu \mathrm{g} / \mathrm{dL})$ and albumin $(3.22 \mathrm{~g} / \mathrm{dl}$; nr. 3.9 4.9 g/dL) were confirmed. Haptoglobin 2-2 $\left(\alpha^{2} \beta\right) 2$ level remained at lower normal level $(26 \mathrm{mg} /$ $\mathrm{dL}$; nr. 15 116 mg/dL). Direct and indirect anti-globulin tests and anti-platelet antibody were positive. Serum levels of creatinine, CEA, CA19-9, SLX, immunoglobulin (Ig) G/A/M, anti-nuclear antibody, rheumatoid factor, anti-thyroglobulin antibody, anti-microsome antibody, C3/C4, anti-mycoplasma antibody and cold agglutination titers were within normal limits. Serologic tests for Helicobacter pylori, human immunodeficiency virus, human T-cell lymphotropic virus type1, hepatitis B virus, hepatitis $C$ virus and syphilis were negative. Serologic tests for Epstein-Barr virus (EBV) showed a prior infection pattern, and IgM antibody against Parvovirus was not detected. A

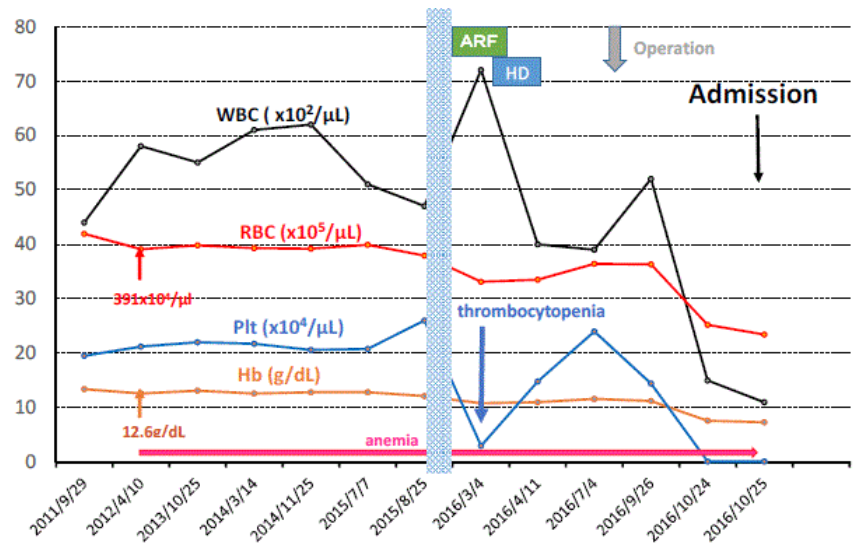

Figure 1. Clinical course before admission. The patient had slight anemia, and it appeared 4 and half years before admission. Acute renal failure (ARF) caused by loxoprofen administration developed in March 2016, but this was improved by hemodialysis (HD). The ARF was associated with remarkable thrombocytopenia (Plt [platelet]: $3 \times 10^{9} / \mathrm{L}$ ). Both disorders were improved by HD. On admission, the remarkable pancytopenia was seen. positron emission computed tomography scan, which was carried out 3 weeks after admission, showed no notable ${ }^{18} \mathrm{~F}$-fluorodeoxyglucose uptake; neither lymphadenopathy nor splenomegaly was present (not shown). The laboratory test results showing hemolysis, positive direct/indirect antiglobulin tests and positive anti-platelet antibody led to a tentative diagnosis of Evans' syndrome. Since the patient concomitantly had remarkable neutropenia, anti-neutrophil antibodies were later examined by using the serum stocked at initial presentation and examined by applying five cell-lineage immunofluorescence test (IFT) [12] and LABScreen MULTI (One Lambda, Inc.) assay [13]. As expected, the patient was found to have anti-neutrophil IgG antibodies specific for human neutrophil alloantigen (HNA)-1a and HNA-2 (Figure 2). Thus, a diagnosis of autoimmune pancytopenia (AIP) was later established.

After admission, administration of prednisolone (PSL) $(40 \mathrm{mg} /$ day) was immediately initiated for 3 days. Although the temperature

(A)

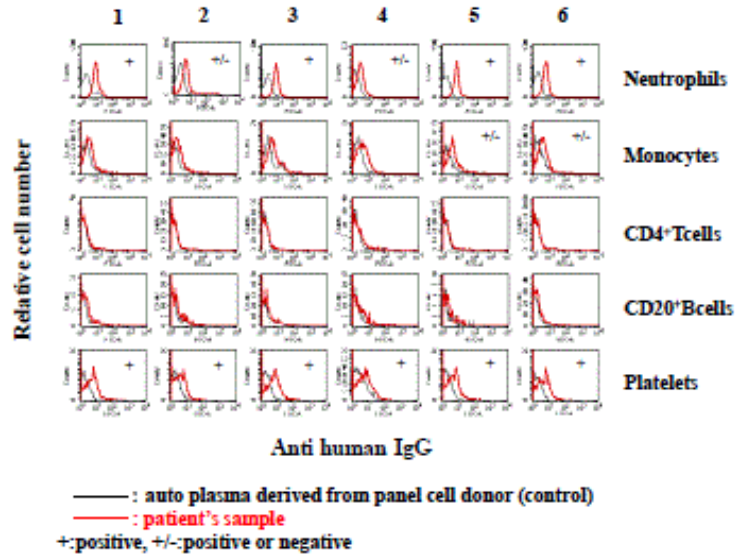

(B)

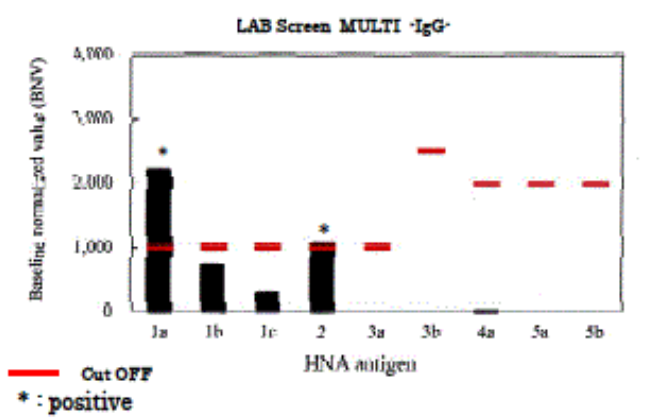

Figure 2. (A) Five cell- lineage IFT showing positive IgG antibodies (asterisk) against neutrophils and platelets. However, the latter positivity was considered to be plateletassociated antibody (possibly anti-platelet IgG antibody) other than HNA, because HNA-1a and HNA-2 are not usually expressed on platelets.

Whole peripheral blood samples were obtained from six healthy volunteers (sample 1-6) and mixed with the patient's serum. Bound antibodies on 5 different blood cell lineages were demonstrated by using fluorescein isethionate-conjugated goat anti-human immunoglobulin G (IgG) and analyzed by flow cytometry. The autologous sera of volunteers were used as negative controls. A positive cut-off was set at more than two times the average fluorescence of negative control of the healthy volunteers.

(B) LABScreen MULTI assay demonstrating anti-neutrophil IgG antibody specific for HNA-1a and HNA-2 (asterisk).

The basis of the LABScreen MULTI is the use of color-coded microbeads coated with HNA peptides. Data acquisition and analysis were performed by the Lambda Assay Beads MULTI Analyte system using the LABScan 100 flow analyzer. 

pathogenesis

dropped rapidly by PSL administration, the pancytopenia was not improved. Then a high-dose methyl-prednisolone (m-PSL) therapy (1000 $\mathrm{mg} /$ day for 3 days with gradual reduction schedule), which was followed by reduced dose of m-PSL and then conventional dose of PSL therapy, was initiated. The steroid therapy was effective; depressed platelet count, neutropenia and elevated CRP level returned to normal 1 week after the initiation of the treatment. The indirect antiglobulin test returned to negative 1 month after the initiation of the steroid therapy, but the direct antiglobulin test remained positive. Anti-platelet antibody became negative about 10 days after admission. In this case, cyclosporine (200 400 mg/day) and metenolone (15 $\mathrm{mg}$ /day) were added to the steroid therapy 2 weeks later because of insufficient recovery from anemia (both drugs were later suspended because of ineffectiveness on anemia). With the reduction of the PSL dose, severe thrombocytopenia associated with positive antiplatelet antibody reappeared 2 months after admission, but this was improved by a semi-high-dose m-PSL (500 mg/day for 3 days) therapy (Figure 3). This event, a febrile episode and elevated levels of CRP, $\beta 2$-microglobulin and s-IL-2R at initial presentation prompted us to investigate the possibility of specific infections by using the stocked serum. Consequently, the patient was found to have cytomegalovirus (CMV) infection; the presence of CMV-IgM antibody as well as CMVDNA was demonstrated in the serum stocked at the reappearance of thrombocytopenia. Serum stocked at initial presentation was retrospectively examined for CMV-IgG and IgM. The results revealed positive CMV-IgG and negative CMV-IgM. Thus, it was considered

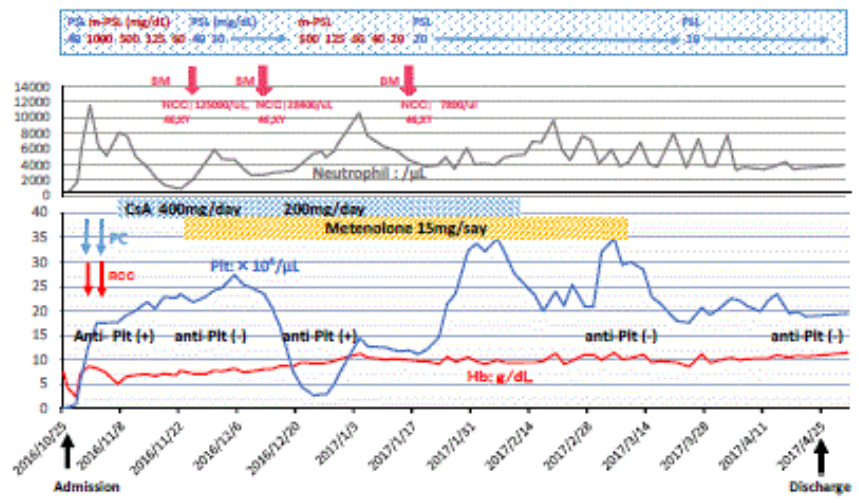

Figure 3. Clinical course after admission. On admission, remarkable pancytopenia was present. After the initiation of steroid therapy, the pancytopenia was immediately improved. However, the improvement of anemia is somewhat insufficient. As reducing the dose of PSL, a transient thrombocytopenia with positive anti-platelet antibody appeared 2 months after admission. Abbreviations: anti-Plt, anti-platelet antibody; BM, bone marrow; CsA, cyclosporine; Hb, hemoglobin; m-PSL, methyl prednisolone; NCC, nuclear cell count; PC, platelet concentrate; Plt, platelet; RCC, red cell concentrate; WBC, white blood cell. that a reactivation of CMV has occurred during the hospital course. The reappeared anti-platelet antibody returned to negative after the recovery from thrombocytopenia (Table 1 and Figure 3).

In the present case, bone marrow (BM) examination could not be done before initiation of the treatment because of patient's exhausted condition, severe anemia and severe thrombocytopenia. Later, the BM aspiration tests were carried out on three occasions during the treatment (November 18, 2106, December 13, 2016 and January 17, 2017). The first examination results revealed erythroid hyperplasia (M/E ratio, 0.5; nr 1.2 3.8) and myelodysplastic features in megakaryocytes and erythroblasts. Micro-megakaryocytes and mononuclear megakaryocytes were frequently seen, and binucleated and trinucleated erythroblasts were sporadically seen. Mature megakaryocytes showing platelet productions were absent. The second and third examinations revealed hypocellular marrows but normal in differential count; however, though very rare, binucleated and trinucleated erythroblasts were seen. Phagocytic findings were absent (Figure 4). Chromosome analyses consistently showed a normal karyotype. Thus, the patient was diagnosed as having refractory anemia (according to the FAB classification of the myelodysplastic syndrome [MDS] [14]), refractory cytopenia with unilineage dysplasia (according to the 2008 revision of the WHO classification [15]) or MDS with single lineage dysplasia (according to the 2016 revision of WHO classification [16]). The patient was discharged on April 22, 2017 and currently remains in good condition (Figure 3); however, CMV-IgM is persistently positive as of May 7, 2017.

\section{Discussion}

For the correct diagnosis of AIP, the demonstration of autoantibodies against three lineages of blood cells is required. The methods to detect autoantibodies against RBCs and platelets are widely used in the clinical field. However, the availability of methods to demonstrate anti-neutrophil antibodies is limited in the clinics. AIN is known as a common form of neutropenia in childhood and is characterized by chronic neutropenia presenting a low absolute neutrophil count of less than $1.5 \times 10 \% / \mathrm{L}$ for more than 6 months [3-6]. This definition of neutropenia is applied also to neutropenia of AIP. Recently, Matsuyama, et al. [12] developed a flow cytometric (FCM) analysis system to detect anti-leukocyte antibodies (i.e., five cell-lineage IFT system). This system made it possible to simultaneously detect antibodies against 5 different individual leukocytes (neutrophils, monocytes, CD4 ${ }^{+} \mathrm{T}$ cells, CD20 ${ }^{+}$ B-cells and platelets). Using this system together with LABScreen microbeads test (i.e., LABScreen MULTI assay system), we were able to demonstrate anti-neutrophil IgG antibody specific for HNA-1a and HNA-2. Thus, it was proved that the patient had autoantibodies for

Table 1. Levels of CMV-IgM/IgG/DNA, autoantibodies, hematologic data, CRP and s-IL-2R during clinical course.

\begin{tabular}{|c|c|c|c|c|c|c|c|}
\hline Date & $10 / 25 / 2016$ & $12 / 2$ & $12 / 24$ & $2 / 7 / 2017$ & $3 / 6$ & $4 / 14$ & $5 / 1$ \\
\hline CMV-IgM $(<0.8$, index $)$ & $<0.8:(-)$ & & 5.01: $(+)$ & 8.9: (+) & 9.2: $(+)$ & 7.83: $(+)$ & 6.77: (+) \\
\hline CMV-IgG $(<2.0$, EIA $)$ & 14.3: $(+)$ & & & & & & 48.7:(+) \\
\hline CMV-DNA $(<100 \mathrm{C} / \mathrm{mL})$ & & & 19200: $(+)$ & & & & \\
\hline Platelet antibody & $(+)$ & $(-)$ & $(+)$ & & $(-)$ & & $(-)$ \\
\hline Direct/indirect AGT & $(+/+)$ & $(+/+)$ & $(+/-)$ & & $( \pm /-)$ & & $(-/-)$ \\
\hline Anti-neutAb & $(+)$ & & & & & & \\
\hline Platelet $\left(\times 10^{9} / \mathrm{L}\right)$ & 1.0 & 24.7 & 2.2 & 34.8 & 34.8 & 23.4 & 12.7 \\
\hline $\mathrm{Hb}(\mathrm{g} / \mathrm{dL})$ & 7.3 & 7.6 & 9.2 & 9.9 & 11.4 & 10.9 & 11.4 \\
\hline Neutrophil $\left(\times 10^{9} / \mathrm{L}\right)$ & 0.42 & 4.71 & 5.39 & 6.80 & 5.92 & 3.70 & 3.92 \\
\hline $\mathrm{CRP}(\mathrm{mg} / \mathrm{dL})$ & 9.73 & 0.20 & 0.02 & 0.05 & 0.05 & 0.02 & 0.02 \\
\hline s-IL-2R (U/L) & 1310 & 1200 & 758 & 591 & 844 & 484 & 393 \\
\hline
\end{tabular}

Abbreviations: AGT, antiglobulin test; Anti-neutAb, anti-neutrophil antibody; EIA, enzyme immune assay, Hb, hemoglobin, CRP, C reactive protein; s-IL-2R, soluble IL-2 receptors. 


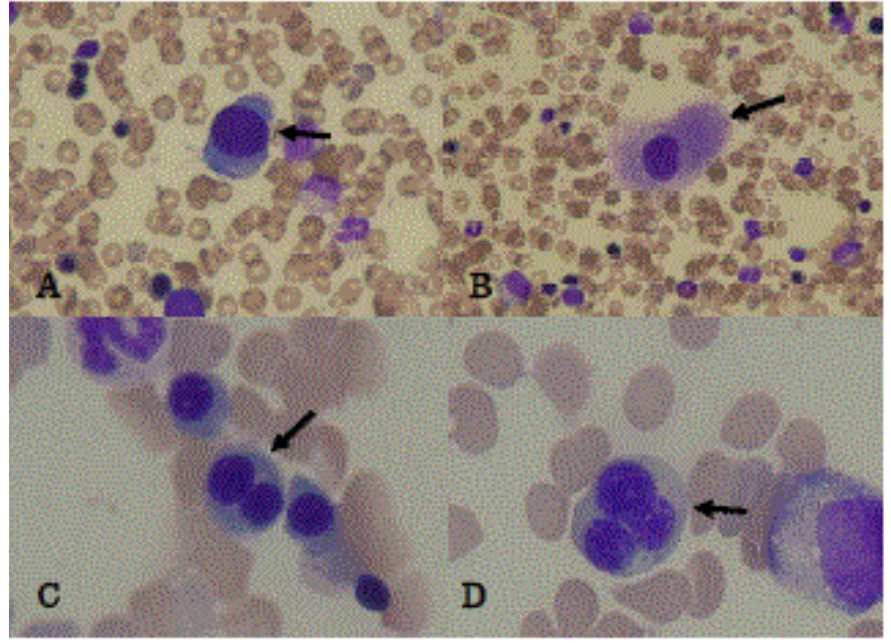

Figure 4. BM smear (May-Grünwald-Giemsa stain, under $\times 40$ magnification objective) (A) A micromegakaryocyte (arrow) seen in the BM obtained on November 18, 2016. (B) A small mononuclear megakaryocyte without platelet production (arrow) seen in the bone marrow obtained on November 18, 2016. Normal mature megakaryocytes were absent in the BM (not shown). (C) A binucleated erythroblast seen in the BM obtained on November 18, 2016. (D) A trinucleated erythroblast (arrow) seen in the BM obtained December 13, 2016 .

three lineages of blood cells, and the diagnosis of AIP was established. Much less is known about idiopathic AIP. Wiesneth, et al. [7] reported 2 cases of idiopathic AIP in 55 cases of unexplained neutropenia, Bux, et al. [5] reported 3 cases of idiopathic AIP in 143 cases of AIN, and Martino, et al. [8] reported 2 cases of idiopathic AIP in 20 cases of AIN, and recently, Mitani, et al. [11] reported 1 case of idiopathic AIP. Thus, 8 cases of idiopathic AIP have been documented in the literature. To establish the diagnosis of idiopathic AIP, it is necessary to completely rule out collagen disease, rheumatoid arthritis, lymphoproliferative disorder, cancer and specific infection causing immune dysfunctions. In the present case, CMV infection was confirmed during the course of illness. In addition, refractory anemia preceded before developing AIP. Thus, this case might represent an aquired AIP due to CMV infection/ reactivation.

Human CMV is a $\beta$ human herpesvirus and can cause lifethreatening infections in immunocompromised patients. It is known that CMV infection can cause thrombocytopenia in immunocompetent adults as well as children [17-25]. In addition, CMV infection is known to trigger an autoimmune process against platelets even though the infection itself is transient and seemingly neither atypical nor severe in nature [19]. CMV infection is also known to cause autoimmune hemolytic anemia [26,27]. In this connection, van Spronsen and Breed [28] reported a case of severe symptomatic thrombocytopenia combined with hemolytic anemia which was caused by CMV infection. Koukoulaki, et al. [29] reported a rare case of a previously healthy woman who developed irreversible pancytopenia secondary to CMV infection in which no autoantibody was detected. To our knowledge, there is no report documenting AIP caused by CMV infection/ reactivation.

In the present case, positive CMV-IgG at initial presentation means that the patient was previously infected quiescently by CMV. The reappearance of thrombocytopenia during the hospital course can be considered to be caused by reactivation of CMV based on the finding of CMV-DNA and CMV-IgM during thrombocytopenia. Since antiplatelet antibody reappeared during thrombocytopenia but returned to negative after improvement of thrombocytopenia, it clearly showed that autoimmune mechanism is involved in developing thrombocytopenia. In the present case, CMV-IgM was negative at initial presentation; however, it should be noted that it takes about 1 week for CMV-IgM to appear in the serum after the beginning of symptoms. In this case, blood sampling was done only 3 days after the beginning of symptoms. Thus, it is conceivable that seroconversion did not yet occurred at initial presentation. Rather, it seems plausible that CMV reactivation had actually occurred at the time of serum sampling. The laboratory data at initial presentation which showed acute inflammatory reactions (e.g., elevated levels of CRP, $\beta 2$ microglobulin and s-IL-2R) and clinical symptoms such as fever and sore throat suggested recent CMV reactivation. It is well known that $s-\mathrm{IL}-2 \mathrm{R}$ will increase via immune activation in cases of viral infection. Thus, CMV reactivation might have also occurred at initial presentation and possibly caused AIP. In the present case, CMV-IgM is still persisting. It is known that in some cases, CMV-IgM may persist for up to 2 years.

Two possible mechanisms have been proposed for the pathogenesis of cytopenia(s) caused by CMV infection: a direct cytopathic effect of CMV on hematopoietic cells and an indirect immune-mediated effect [18]. In the former, several in vitro studies have shown that certain strains of CMV can infect hematopoietic progenitor cells and substantially inhibit hematopoiesis [30,31,32]. In addition, it has been suggested that CMV infection results in downregulation of G-CSF and hematopoietic suppression [31]. Sing and Ruscetti [32] reported that BM cells infected with CMV became totally insensitive to the stimulatory effects of both GM-CSF and G-CSF [31]. Concerning the indirect immune-mediated mechanism, molecular mimicry of CMV has been implicated in the onset of autoimmunity [33]. Viral peptides that share homology with hosts-peptides could initiate an immune response both against itself and the virus. Additionally, during viral cell lysis, intracellular proteins, the secured host antigens not previously recognized, are exposed to circulating antigenpresenting cells that activate autoreactive lymphocytes. In addition, the possibility that hemophagocytic syndrome triggered by CMV can result in the destruction of hematopoietic cells, is also indicated [34]. However, no evidence of hemophagocytosis was found in the bone marrow in our case. Since neutropenia and hemolytic anemia were not exaggerated during the thrombocytopenia reappearance, it seems that megakaryocytic lineage might be more sensitive to CMV infection/ reactivation than myeloid and erythroid lineages.

In the present case, refractory anemia preceded the development of AIP. It is known that autoimmune paraneoplastic syndromes are commonly encountered in patients with MDS. As many as $10 \%$ of MDS patients may experience various autoimmune syndromes [35]. Hemolytic anemia and ITP are known to be seen, though rare, in patients with MDS [35-37]. However, the direct relationship between refractory anemia and AIP seems improbable based upon the patient's clinical course. Rather, it seems probable that AIP simply happened to be complicated by refractory anemia in this case.

Concerning elevated level of IgE at initial presentation, it seems to reflect patient's allergic disposition. This might have been related to previous episode of acute renal failure and thrombocytopenia due to loxoprofen administration. However, this possibility has remained in the realm of surmise due to the lack of clinical data. Furthermore, the relationship between this episode and CMV infection remains unknown.

The distinction between direct and indirect effect of CMV is 

pathogenesis

important because it may have very important therapeutic implications: an immune-mediated effect would be expected to respond to steroids, but a cytopathic effect would not; the direct mechanism presuppose a good response to specific anti-CMV treatments [25]. However, literature review suggested that corticosteroids are also effective in approximately one third of cases [20]. Some physicians believe that treatment with steroids should be avoided in patients with CMV-induced thrombocytopenia because immunosuppressive treatment may cause the exacerbation of primary CMV infection and trigger further decrease in platelet count [24]. If CMV-viremia is demonstrated, viral eradication seems to have a positive influence on the resolution of thrombocytopenia [23]. Treatments with ganciclovir or valgancicloviris is currently recommended as first line treatment for immunocompromised adults with severe CMV disease, and few studies have evaluated the use of these anti-CMV drugs for the treatment in immunocompetent adults [38]. The use of foscarnet, which does not share the myelosuppressive effects of ganciclovir and valganciclovir, seems to be a reasonable first line anti-CMV agent in cases of cytopenia [23]. Splenectomy [21,23,24] and intravenous immunoglobulin $[19,22,24$,$] , useful in patients affected by idiopathic$ thrombocytopenic purpura, are relatively ineffective or refractory for CMV-induced thrombocytopenia. However, it is known that splenectomy regain their efficacy once the primary CMV infection is controlled with anti-CMV agents $[23,24]$. In the present case, anti-viral agents were not used because of remarkable effects of steroid therapy, and because CMV infection/reactivation was not presupposed and retrospectively confirmed after the initiation of steroid therapy. If relapse were to occur in the present case, anti-CMV treatment should be considered. In cases of unexplained acute pancytopenia associated with clinical manifestations of an infectious disease, testing for CMV should be required for better therapeutic consequences.

\section{Conclusions}

A rare case of acquired AIP associated refractory anemia is documented. In particular, the recognition of AIP should be requisite to some cases of Evans' syndrome associated with leukocytopenia. Special consideration should be given to the possible involvement of CMV infection in pathogenesis of immune cytopenia(s) or AIP. We emphasize the importance of testing for anti-neutrophil antibody in cases of pancytopenia and AIP. Insufficient recovery from anemia in the present case seemed to be related to coexisting refractory anemia.

\section{Acknowledgements}

The authors would like to thank Nobuki Matsuyama (medical antineutrophil antibody and providing the data, and Prof. Hong-Kean Ooi (Department of Veterinary Medicine, Azabu University) for helpful reviewing and preparing the manuscript.

\section{Consent}

Written informed consent was obtained from the patient for publication of this case report and accompanying images.

\section{Competing interests}

The authors declare no competing interests.

\section{References}

1. Evans RS, Duane RT (1949) Acquired hemolytic anemia; the relation of erythrocyte antibody production to activity of the disease, the significance of thrombocytopenia and leukopenia. Blood 4: 1196-1213.
2. Evans RS, Takahashi K, Duane RT, Payne R, LIU C (1951) Primary thrombocytopenic purpura and acquired hemolytic anemia; evidence for a common etiology. AMA Arch Intern Med 87: 48-65. [Crossref]

3. Boxer LA, Greenberg MS, Boxer GJ, Stossel TP (1975) Autoimmune neutropenia. N Engl J Med 293: 748-753. [Crossref]

4. Lalezari P, Jiang AF, Yegen L, Santorineou M (1975) Chronic autoimmune neutropenia due to anti-NA2 antibody. N Engl J Med 293: 744-747.

5. Bux J, Kissel K, Nowak K, Spengel U, Mueller-Eckhardt C (1991) Autoimmune neutropenia: clinical and laboratory studies in 143 patients. Ann Hematol 63: 249-252.

6. Nakamura K, Miki M, Mizoguchi Y, Karakawa S, Sato T, et al. (2009) Deficiency of regulatory $\mathrm{T}$ cells in children with autoimmune neutropenia. Br J Haematol 145: 642-647. [Crossref]

7. Wiesneth M, Pflieger H, Frickhofen N, Heimpel H (1985) Idiopathic combined immunocytopenia. Br J Haematol 61: 339-348. [Crossref]

8. Martino R, Muñiz-Díaz E, Arilla M, Ibáñez M, Altés A, et al. (1995) Combined autoimmune cytopenias. Haematologica 80: 305-310. [Crossref]

9. Klumpp TR, Caliguri MA, Rabinowe SN, Soiffer RJ, Murray C, et al. (1990) Autoimmune pancytopenia following allogeneic bone marrow transplantation. Bone Marrow Transplant 6: 445-447.

10. De Lord C, Marsh JCW, Smith JG, Singer CRJ, Gordon-Smith EC (1996) Fata autoimmune pancytopenia following bone marrow transplantation for aplastic anaemia. Bone Marrow Transplant 18: 237-239.

11. Mitani S, Okubo Y, Nishi K, Takahashi S, Tada K, et al. (2016) Rapidly progressive autoimmune pancytopenia successfully treated with steroids. Rinsho Ketsueki 57: 2324-2328. [Crossref]

12. Matsuyama N, Kojima Y, Hirayama F, Yasui K, Taniue A, et al (2006) Simultaneous five cell-lineage flow cytometric analysis system for detection of leucocyte antibodies. Transfusion Medicine 16: 111-118.

13. Schulz U, Reil A, Kiefel V, Bux J, Moog R (2017) Evaluation of a new microbeads assay for granulocyte antibody detection. Transfusion 57:70-81.

14. Bennett JM, Catovsky D, Daniel MT, Flandrin G, Galton DA, et al. (1982) Proposals for the classification of the myelodysplastic syndromes. Br J Haematol 51: 189-199. [Crossref]

15. Vardiman JW, Thiele J, Arber DA, Brunning RD, Borowitz MJ, et al. (2009) The 2008 revision of the World Health Organization (WHO) classification of myeloid neoplasms and acute leukemia: rationale and important changes. Blood 114: 937-951.

16. Arber DA, Orazi A, Hasserjian R, Thiele J, Borowitz MJ, et al (2016) The 2016 revision to the World Health Organization classification of myeloid neoplasms and acute leukemia. Blood 127: 2391-2405.

17. Sissons JG, Carmichael AJ (2002) Clinical aspects and management of cytomegalovirus infection. J Infect 44: 78-83. [Crossref]

18. Eisenberg MJ, Kaplan B (1993) Cytomegalovirus-induced thrombocytopenia in an immunocompetent adult. West J Med 158: 525-526. [Crossref]

19. DiMaggio D, Anderson A, Bussel JB (2009) Cytomegalovirus can make immune thrombocytopenic purpura refractory. Br J Haematol 146: 104-112. [Crossref]

20. Noguchi M, Aruga S, Kato A, Oshimi K (2000) Cytomegalovirus mononucleosis with severe thrombocytopenia. Rinsho Ketueki 41: 1171-1177.

21. Alliot C, Barrios M (2005) Cytomegalovirus-induced thrombocytopenia in an immuncompetent adult effectively treated with intravenous immunoglobulin: a case report and review. Hematology 10: 277-279.

22. Flores-Chang BS, Arias-Morales CE, Wadskier FG, Gupta S, Stoicea N (2015) Immune thrombocytopenic purpura secondary to cytomegalovirus infection: a case report. Front Med (Lausanne) 2: 79.

23. Yaari S, Koslowsky B, Wolf D, Chajek-Shaul T, Hershcovici T (2010) CMV-related thrombocytopenia treated with foscarnet: a case series and review of the literature. Platelets 21: 490-495.

24. Shrestha R, Rondelli D, Sherpa MT (2014) Cytomegalovirus:a possible cause of persistent refractory immune thrombocytopenic purpura. J Adv Intern Med 3: 42-45.

25. Palumbo E, Bonora G (2008) Cytomegalovirus-induced thrombocytopenia in an immunocompetent child. Infect Dis Clin Practice 16: 54-56.

26. Salloum E, Lundberg WB (1994) Hemolytic anemia with positive direct antiglobulin test secondary to spontaneous cytomegalovirus infection in healthy adults. Acta 
Takemori N (2017) A rare case of autoimmune pancytopenia (AIP) preceded by refractory anemia: possible involvement of cytomegalovirus (CMV) infection in pathogenesis

\section{Haematol 92: 39-41}

27. Taglietti F, Drapeau CM, Grill E, Capone A, Noto P, et al. (2010) Hemolytic anemia due to acute cytomegalovirus infection in an immunocompetent adult: a case report and review of the literature. J Med Case Reports 4: 334

28. van Spronsen DJ, Breed WP (1996) Cytomegalovirus-induced thrombocytopenia and haemolysis in an immunocompetent adult. Br J Haematol 92: 218-220. [Crossref]

29. Koukoulaki M, Ifanti G, Grispou E, Papastamopoulos V, Chroni G, et al. (2010) Fulminant pancytopenia due to cytomegalovirus infection in an immunocompetent adult. Braz J infect Dis 14.

30. Maciejewski JP, Bruening EE, Donahue RE, Mocarski ES, Young NS, et al. (1992) Infection oh hematopoietic progenitor cells by human cytomegalovirus. Blood 80: 170-178.

31. Simmons P, Kaushansky K, Torok-Storb B (1990) Mechanisms of cytomegalovirusmediated myelosuppression: perturbation of stromal cell function versus direct infection of myeloid cells. Proc Natl Acad Sci USA 87: 1386-1390.

32. Sing GK, Ruscetti FW (1990) Preferential suppression of myelopoiesis in normal human bone marrow cells after in vitro challenge with human cytomegalovirus. Blood 75: $1965-1973$

33. Panoutsakopoulou V, Sanchirico ME, Huster KM, Jansson M, Granucci F, et al. (2001)
Analysis of the relationship between viral infection and autoimmune disease. Immunity 15: $137-147$.

34. Ina S, Tani M, Takifuji K, Yamazoe S, Nakatani Y, et al. (2004) Virusassociated hemophagocytic syndrome and hemorrhagic jejunal ulcer caused by cytomegalovirus infection in a noncompromised host; a case report of unusual entity. Hepatogastroenterology 51: 491-493.

35. Saif MW, Hopkins JL, Gore SD (2002) Autoimmune phenomena in patients with myelodysplastic syndromes and chronic myelomonocytic leukemia. Leuk Lymphoma 43: 2083-2092.

36. Enright H, Miller W (1997) Autoimmune phenomena in patients with myelodysplastic syndromes. Leuk Lymphoma 24: 483-489. [Crossref]

37. Takezako N, Shikai T, Tanimura A, Inokuchi C, Yamagata N, et al. (2003) [Myelodysplastic syndrome accompanied by Evans syndrome]. Rinsho Ketsueki 44: 396-400. [Crossref]

38. Lancini D, Faddy HM, Flower R, Hogan C (2014) Cytomegalovirus disease in immunocompetent adults. Med J Aust 201: 578-580. [Crossref]

Copyright: $\odot 2017$ Takemori N. This is an open-access article distributed under the terms of the Creative Commons Attribution License, which permits unrestricted use, distribution, and reproduction in any medium, provided the original author and source are credited. 